Research Paper

\title{
Exosomal ephrinA2 derived from serum as a potential biomarker for prostate cancer
}

\author{
Shibao Li1 ${ }^{1,}{ }^{, *}$, Yao Zhao ${ }^{1, *}$, Wenbai Chen ${ }^{3,}$, , Lingyu Yin ${ }^{1}$, Jie Zhu ${ }^{1}$, Haoliang Zhang ${ }^{1}$, Chenchen Cai ${ }^{1}$, \\ Pengpeng $\mathrm{Li}^{2}$, Lingyan Huang ${ }^{1}$, Ping $\mathrm{Ma}^{1,2}{ }^{\square}$ \\ 1. Medical Technology Institute of Xuzhou Medical University, Xuzhou 221004, China \\ 2. Department of Laboratory Medicine, Affiliated Hospital of Xuzhou Medical University, Xuzhou 221002, China. \\ 3. Department of Nuclear Medicine Laboratory, Affiliated Hospital of Xuzhou Medical University, Xuzhou 221002, China. \\ * These authors contributed equally to this work. \\ $\triangle$ Corresponding author: Ping Ma, Professor, Department of Laboratory Medicine, Affiliated Hospital of Xuzhou Medical University, 99 West Huaihai Road, \\ Xuzhou, 221002, China. E-mail: pingm62@aliyun.com \\ (c) Ivyspring International Publisher. This is an open access article distributed under the terms of the Creative Commons Attribution (CC BY-NC) license \\ (https:// creativecommons.org/licenses/by-nc/4.0/). See http://ivyspring.com/terms for full terms and conditions.
}

Received: 2018.01.29; Accepted: 2018.05.03; Published: 2018.06.23

\begin{abstract}
Up-regulation of serum ephrinA2 is common in various malignancies and has been suggested as a potential biomarker for the diagnosis and prognosis of prostate cancer (PCa). However, the type of serum ephrinA2 expressed in PCa patients remains elusive. Furthermore, the level of exosomal ephrinA2 derived from serum is increased in patients with osteoporosis, a common complication of $\mathrm{PCa}$ patients undergoing androgen deprivation therapy. It is unknown whether exosomes derived from PCa patient serum contains ephrinA2. In this study, we explored the ephrinA2 expression in whole serum and tissues and identified the circulating exosomal ephrinA2 as a potential biomarker for PCa. Exosomes were isolated from patient sera by differential centrifugation and the presence of ephrinA2 was confirmed via electron microscopy and western blotting. The type of ephrinA2 in serum was evaluated by western blotting. The expression of serum ephrinA2 including secreted and cleaved ephrinA2 and exosomal ephrinA2 were detected by ELISA and western blotting. Compared with benign prostatic hyperplasia (BPH) and controls, the levels of whole serum ephrinA2 and exosomal ephrinA2 were significantly higher in PCa patients. Moreover, exosomal ephrinA2 expression was positively correlated with TNM staging and Gleason score of PCa patients. The diagnostic efficiency of exosomal ephrinA2 was superior to that of whole serum ephrinA2 and serum PSA in distinguishing PCa patients from those from BPH patents. Our study indicates that exosomal ephrinA2 has high potential as a biomarker for the presence of $\mathrm{PCa}$ and offers a new therapeutic target for this disease.
\end{abstract}

Key words: EphrinA2, Prostate cancer, Exosomal ephrinA2, Gleason score, TNM staging.

\section{Introduction}

Prostate cancer (PCa) is the most common malignancy worldwide ${ }^{[1]}$. Until now, PSA screening is widely used in detecting PCa. However, PSA is an organ-specific marker rather than PCa-specific biomarker which makes PSA testing an excessive examination procedure and requires interventional therapy[2, 3]. Therefore, sensitive and specific biomarkers for $\mathrm{PCa}$ diagnosis and prognosis are urgently needed.
EphrinA2, located on chromosome 19p13.3, is a ligand to several Eph receptors including EphA3, EphA4, EphA5, and EphA8[4]. EphrinA2, is a membrane-bound protein with a glycosylphosphatidylinositol (GPI) lipid anchor. It has been implicated in various physiological processes such as tissue and organ development, neovascularization, cell adhesion and separation[5], and also in the progression of many disease including oncogenesis. For example, Fox et al.[6] demonstrated by 
identification of Eph/ephrin profiles that ephrinA2 is involved in tumor invasiveness and tumorigenesis. Feng et al.[7] systematically evaluated the role of ephrinA2 in hepatocellular carcinoma (HCC) and found that ephrinA2 is significantly up-regulated both in cell lines and clinical tissue samples of HCC, which mainly contributed to apoptosis rather than proliferation in the progression of HCC. Several studies showed that ephrinA2 transcript is overexpressed in breast cancer and renal cell carcinoma, implying that ephrinA2 may be a potential biomarker for these cancer ${ }^{[6,8]}$. Our previous studies also showed that the level of circulating ephrinA2 is significantly higher in patients with prostate cancer than those with benign prostatic hyperplasia $(\mathrm{BPH})$ or health controls ${ }^{[9]}$. Furthermore, a recent study reported that exosomal ephrinA2 derived from serum was up-regulated in patients with osteoporosis[10], a common complication of PCa patients undergoing androgen deprivation therapy[11]. The data above suggests that ephrinA2 may be a potential biomarker for the diagnosis or prognosis of many diseases. Yet, the expression of exosomal ephrinA2 in PCa remains elusive.

In this study, we initially explored the ephrinA2 expression in whole serum and tissues. Subsequently, we analyzed for the circulating exosomal ephrinA2 in clinical samples and evaluated the correlation between exosomal ephrinA2 and tumor-nodemetastasis (TNM) staging and Gleason score. Finally, we investigated the diagnostic efficiency of exosomal ephrinA2 for $\mathrm{BPH}$ and $\mathrm{PCa}$ patients.

\section{Materials and Methods}

\section{Clinical samples}

Serum samples were collected from 20 healthy persons (controls), $21 \mathrm{BPH}$ patients, and $50 \mathrm{PCa}$ patients (Table 1). Blood samples were centrifuged at $3000 \mathrm{~g}$ for $5 \mathrm{~min}$ to extract serum which was stored at $-80^{\circ} \mathrm{C}$ until analyzed. Each sample was divided into two parts, one for ELISA test, and another for exosome isolation. All tissue specimens were obtained from patients undergoing prostate needle biopsies (PNB) or radical prostatectomy (RPE). A written consent was obtained from each patient.

\section{Exosome isolation}

Exosomes were isolated with a minor modified protocol according to Thery et al[12]. Briefly, thawed, serum samples $(400 \mu l)$ diluted in PBS were centrifuged for $30 \mathrm{~min}$ at $2000 \times \mathrm{g}$ and for $45 \mathrm{~min}$ at $12000 \times \mathrm{g}$. Afterwards, the samples were filtered through a $0.22-\mu \mathrm{m}$ pore filter (Millipore, Bedford, USA), followed by ultracentrifugation (Beckman Coulter, Inc. CA, USA) at $150000 \mathrm{~g}$ for $2 \mathrm{~h}$. Thereafter, the supernatant was collected and designated as exosome-depleted supernatant (Sn), and the pellets were washed followed by a second step of ultracentrifugation at $150,000 \times \mathrm{g}$ for $2 \mathrm{~h}$ and re-suspended in $80 \mu \mathrm{l}$ lysis buffer. The amount of pellet protein was measured by the BCA protein assay kit (Beyotime, Nantong, China). These pellets were divided into aliquots and kept at $-80^{\circ} \mathrm{C}$ until further analysis.

\section{Transmission Electron microscopy (TEM)}

A drop of exosome pellets (approximately 20 $\mathrm{\mu l}$ ) was placed on the formvar carbon-coated nickel grid for $5 \mathrm{~min}$. Then, a drop of uranyl acetate was placed on the grid and incubated for $1 \mathrm{~min}$ in the dark. After standing for $30 \mathrm{~min}$, the grids were examined with TEM (Hillsboro, FEI, USA).

\section{Western blotting}

Western blotting procedures were performed following a standard protocol. The protein was solubilized with loading buffer $(5 \times)$ and heated at $100^{\circ} \mathrm{C}$ for $10 \mathrm{~min}$. Proteins were separated by SDS-PAGE using 10\% gels and transferred to a polyvinylidene fluoride membrane (PVDF, Bio-Rad Laboratories Inc, California, USA). The membranes were incubated overnight at $4^{\circ} \mathrm{C}$ with primary antibodies: rabbit anti-human CD63 (1:500, SBI, USA), rabbit anti-human $\beta$-actin (1:5000, Proteintech, USA), mouse anti-human GAPDH (1:2000, Proteintech, USA), and rabbit anti-human ephrinA2 (1:200, DF3027, Affinity Biosciences, USA) followed by horseradish peroxidase-conjugated secondary anti-rabbit and anti-mouse (1:2000, CoWin Biosciences, China) for $2 \mathrm{~h}$. The membranes were visualized with enhanced chemiluminescent substrate (Bio-Rad Laboratories Inc, California, USA).

\section{ELISA}

Both serum EphrinA2 and exosomal EphrinA2 were quantified using the ELISA kit (TSZ, NJ, USA) according to the manufacturer's instructions. Briefly, concentration gradient of calibrators, blank controls, and sample were placed into the microwells. Thereafter, the plates were incubated for $30 \mathrm{~min}$ at 37 ${ }^{\circ} \mathrm{C}$ and washed five times with washing buffer. Then, horseradish peroxidase (HRP)-conjugated antihuman ephrinA2 antibody was added and incubated for $30 \mathrm{~min}$ at $37^{\circ} \mathrm{C}$. After washing, $50 \mu \mathrm{l}$ tetramethylbenzidine (TMB) substrate was added and incubated for $20 \mathrm{~min}$ at $37^{\circ} \mathrm{C}$ in the dark. Finally, a stop solution was added to each well, and incubated for $10 \mathrm{~min}$. The absorbance of the resulting product was read at $450 \mathrm{~nm}$ within $15 \mathrm{~min}$ via the microplate reader (Bio-Rad Laboratories Inc, California, USA). All samples were assessed in duplicate. 


\section{Statistical analysis}

All statistical analyses were performed using SPSS16.0 (SPSS, Chicago, USA). A two-sided $P<0.05$ was considered statistically significant. The one-way analysis of variance (ANOVA) was used to evaluate the statistical significance among patient groups. Receiver operating characteristic (ROC) analysis was used to verify whether exosomal ephrinA2 and serum PSA levels could be used to distinguish patients with PCa from BPH patients.

\section{Result}

\section{Tissue and circulating level of ephrinA2 were up-regulation in $\mathbf{P C a}$}

To investigate the expression profile of ephrinA2 in clinical samples, we first analyzed ephrinA2 protein expression in tissues via western blotting in 9 paired prostate carcinomas and noncancerous tissues. The result showed that the expression of ephrinA2 protein was relatively higher in PCa tissues (Figure 1A). We thereafter detected whole serum ephrinA2 concentrations by ELISA in 20 heath controls, $21 \mathrm{BPH}$, and $50 \mathrm{PCa}$ patients (Table 1). Consistent with our previous studies [9], serum ephrinA2 concentrations were significantly higher in PCa patients, compared to $\mathrm{BPH}$ patients and controls (Figure $1 \mathrm{~B}, P<0.001$ ).
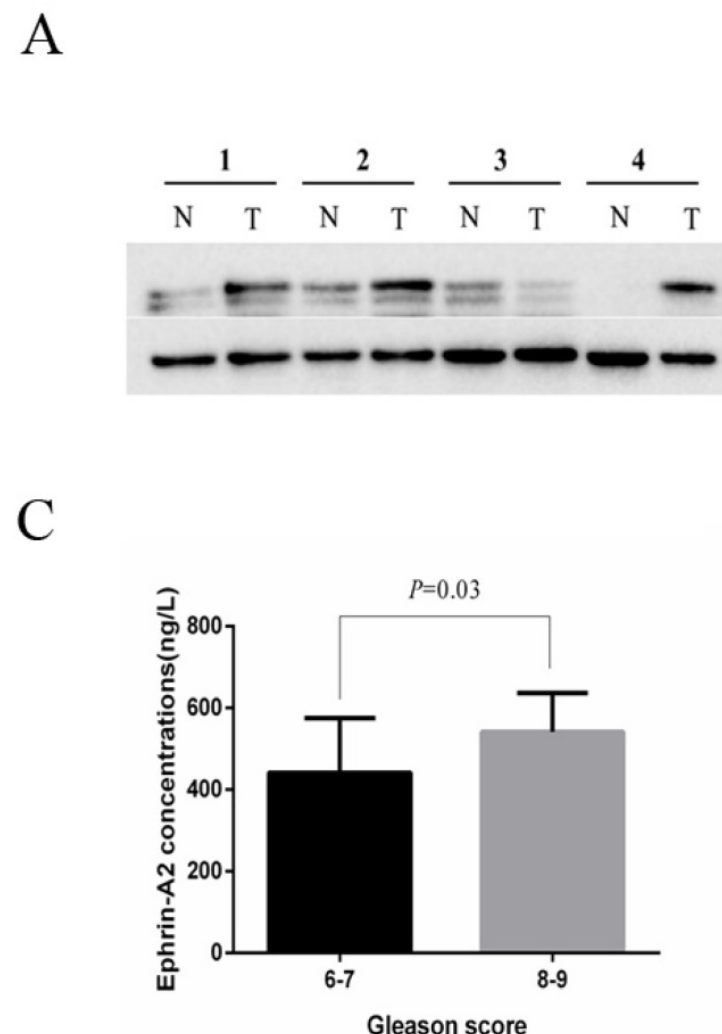

Furthermore, ephrinA2 was positively correlated with Gleason score $(P=0.03)$ (Figure $1 C)$. However, there was no correlation between whole serum ephrinA2 and patient's age, PSA levels and TNM (Figure 1C).

\section{Characterization of circulating exosomes}

Exosomes isolated from serum were observed by TEM. As shown in Figure 2A, exosomal ephrinA2 had a rounded morphology with a size of approximately 100nm. The expression of CD63, a marker for exosomes, confirmed the presence of exosomes (Figure 2B).

\section{Identification of ephrinA2 on serum derived-exosomes}

To verify whether the circulating exosomes contained ephrinA2, Sn and exosomes were evaluated by western blotting. $\beta$-actin was used to maintain western blotting loading. As expected, the result showed that ephrinA2 was detected both in exosomal fractions and Sn (Figure 3). The exosomal ephrinA2 had a small part of the whole which was consistent with ELISA results.

\section{Exosomal ephrinA2 was up-regulated in PCa}

To explore the prevalence of exosomal ephrinA2 in $\mathrm{PCa}$, we analyzed its expression by ELISA in 20
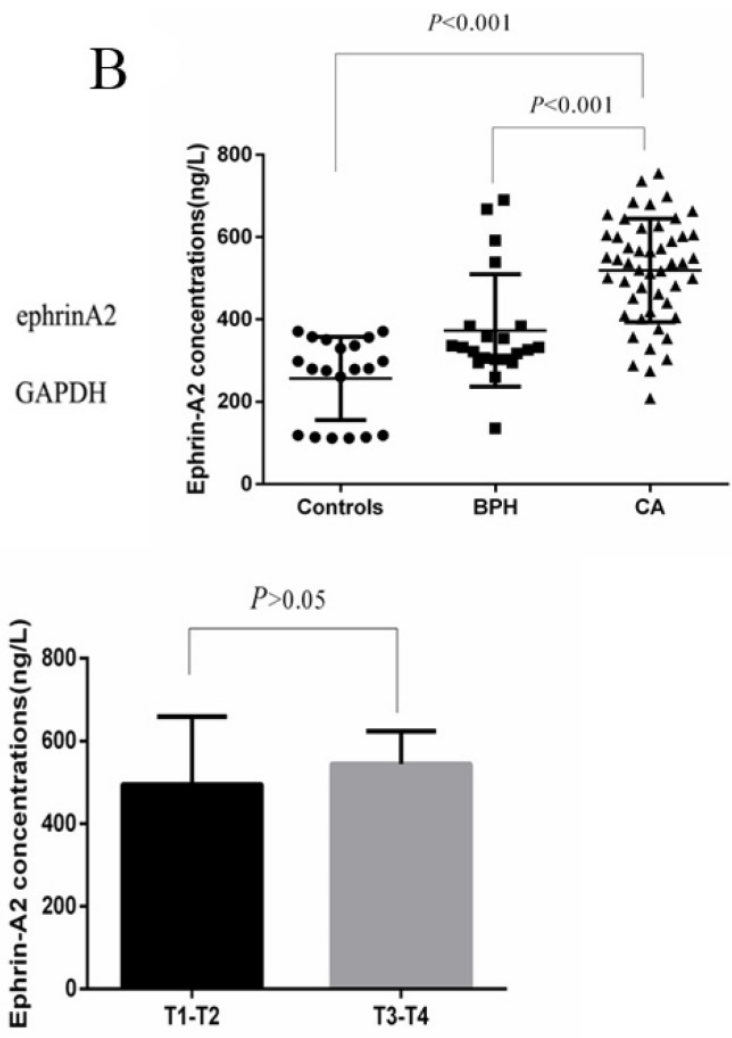

Fig. 1. Expression of ephrinA2 in tissues and serum. A. Expression of ephrin A2 protein in 9 pairs of PCa tissues by western blotting (N, adjacent tissue; $\mathrm{T}$, tumor tissue). The result shows the representative western blots in four pairs. B. Expression of ephrinA2 protein in 20 healthy controls, 21 BPH patients and 50 PCa patients measured via ELISA. C. The correlation between exosomal ephrinA2 and TNM staging or Gleason score respectively. 
healthy controls, $21 \mathrm{BPH}$, and 50 PCa patients (Table 1). Compared to controls and $\mathrm{BPH}$, the concentrations of ephrinA2 were significantly higher in PCa patients (Figure 4A), which was in agreement with the western blotting results (Figure 4B). Furthermore, ephrinA2 expression was positively correlated with Gleason score $(P=0.02)$ and TNM $(P=0.03)$ (Figure $4 C)$. But, there was no correlation between exosomal ephrinA2 and patient's age, and PSA levels (Table 1).

Table 1. Correlations between exosomal ephrinA2 levels and clinicpathological parameters.

\begin{tabular}{|c|c|c|c|c|}
\hline & Prostate cancer & $\mathrm{BPH}$ & $\begin{array}{l}\text { Health } \\
\text { Persons }\end{array}$ & $P$ \\
\hline Case, $\mathrm{n}$ & 50 & 21 & 20 & \\
\hline $\begin{array}{l}\text { Age (mean } \pm S D \text {, } \\
\text { years) }\end{array}$ & $72.2 \pm 8.2$ & $71.5 \pm 9.6$ & $72.0 \pm 9.6$ & 0.95 \\
\hline \multicolumn{5}{|l|}{ PSA (ng/ml) } \\
\hline $4-10$ & 10 & 8 & & 0.12 \\
\hline$>10$ & 40 & 13 & & \\
\hline \multicolumn{5}{|l|}{ TNM staging } \\
\hline $\mathrm{T} 1-\mathrm{T} 2$ & 18 & & & 0.03 \\
\hline T3-T4 & 32 & & & \\
\hline \multicolumn{5}{|l|}{ Gleason score } \\
\hline $6-7$ & 19 & & & 0.02 \\
\hline $8-9$ & 31 & & & \\
\hline
\end{tabular}

\section{Evaluation of diagnostic efficiency}

To distinguish PCa from BPH patients, ROC curve was performed based on the ephrinA2 levels and PSA. The whole serum ephrinA2 levels were superior in differentiating $\mathrm{PCa}$ patients from those with $\mathrm{BPH}$ (area under curve (AUC) $=0.7666$; confidence interval $(\mathrm{CI})=0.6284-0.9047 ; \quad P=0.0004)$, to whole serum PSA levels (AUC $=0.6043 ; \mathrm{CI}=0.4586-0.7499$; $P=0.1678$ ) (Figure $5 \mathrm{~A}$ ). The optimal cut-off point of ephrinA2 concentration was $392.5 \mathrm{ng} / \mathrm{L}$, with a sensitivity of $80.39 \%$ and a specificity of $80.59 \%$, whereas the whole serum PSA cutoff value was 38.43 , with a sensitivity of $42.86 \%$ and a specificity of $82 \%$ (Figure 5C). The exosomal ephrinA2 levels were superior in differentiating $\mathrm{PCa}$ patients from those BPH (AUC=0.9062; CI=0.8191-0.9933; $P<0.0001)$, than PSA levels (Figure 5B). The optimal cut-off points of exosomal ephrinA2 concentration was $20.4 \mathrm{ng} / \mathrm{L}$, with a sensitivity of $88 \%$ and a specificity of $80.95 \%$.

\section{Discussion}

In this study, we demonstrated that the level of ephrinA2 was up-regulated both in serum and in tissues from PCa patients. Similarly, exosomal ephrin A2 was significantly higher in PCa patients and it was positively correlated with Gleason score and TNM staging. Furthermore, based on the ROC curve of two different forms of ephrinA2, we found that exosomal ephrinA2 was superior to the whole serum ephrinA2 and PSA in distinguishing PCa patients from those with $\mathrm{BPH}$ which suggested that exosomal ephrinA2 may be a better biomarker for the diagnosis and prognosis of $\mathrm{PCa}$.
A

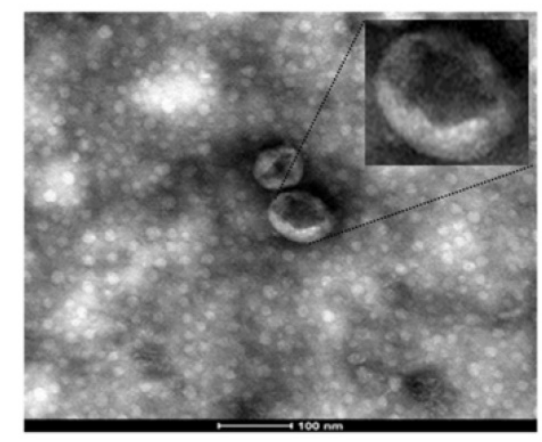

B

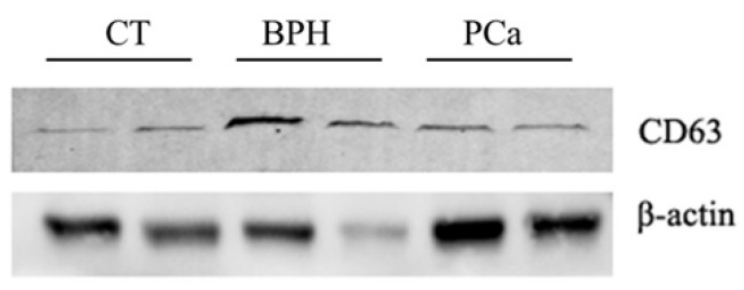

Fig. 2. Confirmation of the ultracentrifugation pellets containing exosomes. A. TEM micrographs of exosome isolation by ultracentrifugation. Bar represents $100 \mathrm{~nm}$. B. Western blotting for exosomes using exosomal marker CD63.

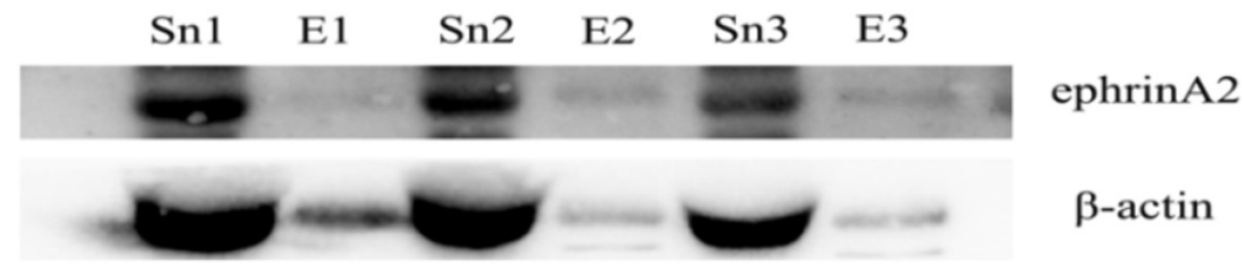

Fig. 3. Expression of ephrinA2 in exosomes and exosome-depleted supernatant respectively. E, exosomes. Sn, exosome-depleted supernatant. 
A

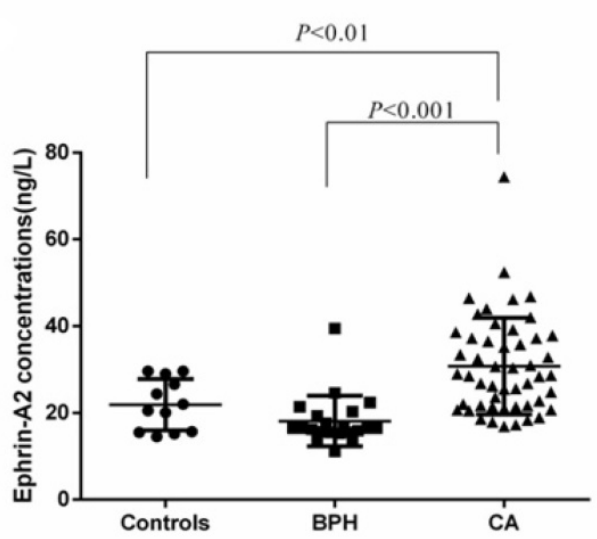

B
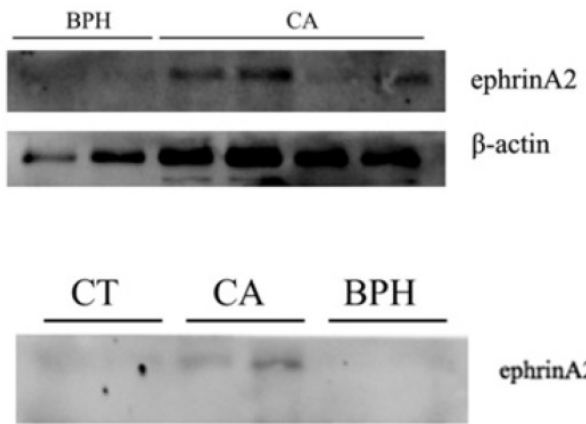

ephrinA2

$\beta$-actin

$\mathrm{C}$
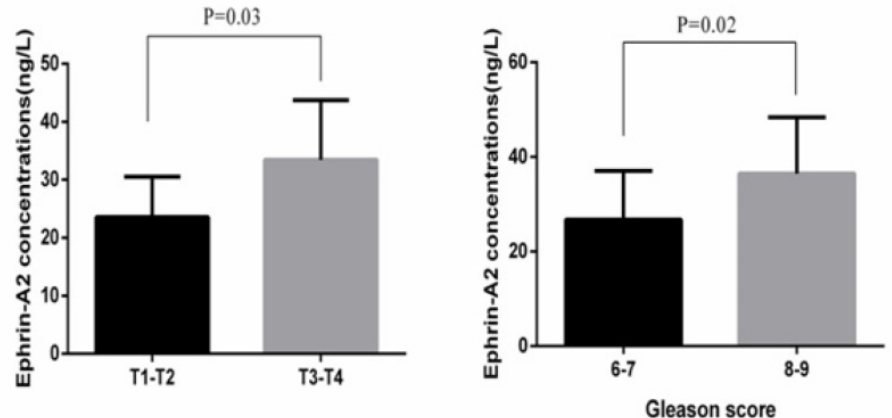

Fig. 4. Exosomal ephrinA2 expression in clinical samples. A. Expression of ephrinA2 protein in 20 healthy controls, $21 \mathrm{BPH}$ patients and $50 \mathrm{PCa}$ patients was measured via ELISA. B. The correlation between exosomal ephrinA2 and TNM staging or Gleason score respectively. C. Expression of exosomal ephrinA2 in patients evaluated by western blotting.

A

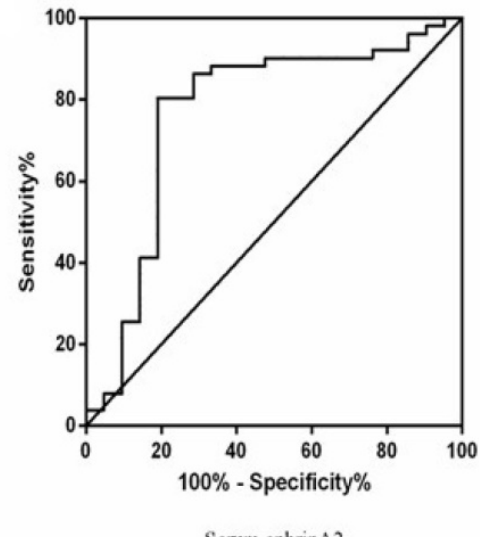

B

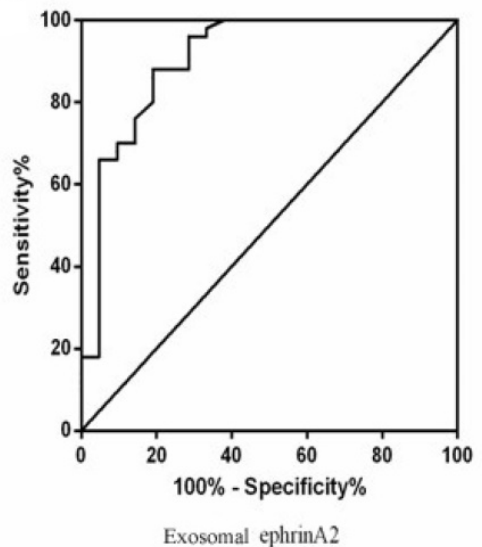

$\mathrm{C}$

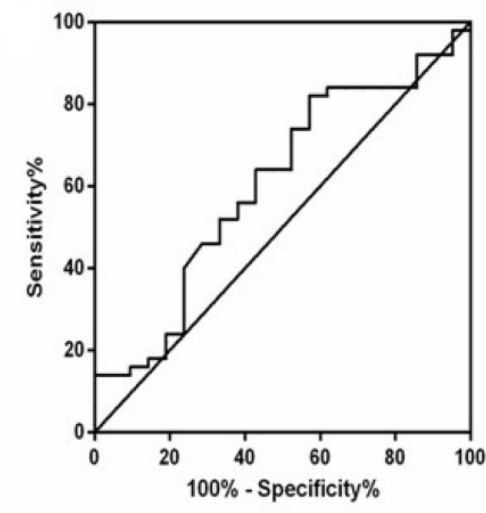

PSA

Fig. 5. ROC curve for ephrinA2 and serum PSA. A. ROC curve analysis of serum ephrinA2 expression in BPH patients versus PCa patients with a specificity of $80.39 \%$, and a sensitivity of $80.59 \%$. B. ROC curve analysis of exosomal ephrinA2 expression in BPH patients versus PCa patients with a specificity of $88 \%$ and a sensitivity of $80.59 \%$. C. ROC curve analysis of serum PSA expression in BPH patients versus PCa patients with a specificity of $42.8 \%$, and a sensitivity of $82 \%$.

Typically, exosomes are secreted by all cells with a diameter of 30-120nm lipid bilayer membrane and they can be identified in biological fluids including urine, blood, serum and saliva ${ }^{[13-17]}$. Exosomes are small extracellular vesicles with various molecular constituents including proteins, mRNA and microRNA which have the potential to be biomarkers in various malignancies including $\mathrm{PCa}^{[18]}$. Increasing evidence has demonstrated that most of Eph receptors and ephrin ligands can be detected in exosomes. For example, proteomic profiling has identified $11 \mathrm{Eph}$ receptors and 2 ligands (ephrinB1, ephrinB2) in colon cancer cell-derived exosomes ${ }^{[19]}$. In addition, Gong[20] revealed that EphB2 anchored on the exosomes can induce ephrinB1 reverse signaling and bring about neuronal axon repulsion. Furthermore, a study reported that ephrinA2 was identified in exosomes which were derived from serum of osteoporosis patients ${ }^{[10]}$, a complication of PCa patients undergoing androgen deprivation therapy[11], which implied that exosomal ephrinA2 may play an important role in PCa disease progression. In the present study, we not 
only found the presence of ephrinA2 in exosomes purified from serum of PCa patients, but also observed that the level of serum exosomal ephrinA2 was significantly higher in PCa patients than in $\mathrm{BPH}$ patients and controls. Moreover, a significant association between exosomal ephrinA2 and clinicopathologic TNM staging and Gleason score was observed in this cohort study. These results implied that exosomal ephrinA2 may be a useful serum marker for the diagnosis and prognosis of PCa.

We further analyzed the ephrinA2 expression in 9 paired prostate carcinomas and noncancerous tissues. In line with the elevated serum exosomal ephrinA2 in PCa patients, the expression of ephrinA2 protein was relatively higher in $\mathrm{PCa}$ tissues than noncancerous tissues which is consistent with previous studies ${ }^{[9]}$. Moreover, the number of exosomes in serum derived from PCa patients was more than that of noncancerous patients[21]. The above data suggests that prostatic tumor cells may produce more exosomes expressing ephrinA2.

Interestingly, other soluble isoforms of ephrinA2 have also been identified in serum from PCa patients. Being a membrane-bound protein with a GPI lipid anchor, how is ephrinA2 released into the blood? Hattori et al. ${ }^{[22]}$ confirmed that the membrane-bound ephrinA2 may be a substrate of ADAM10, a transmembrane metalloprotease shedding a range of cell surface proteins including ephrinA ligands, which releases soluble fragments into the cell medium. Since the activity of ADAM10 is elevated in PCa tissues[23], a possible reason for the elevated serum ephrinA2 concentration is the cleavage of ephrinA2 at the prostate tumor tissue by ADAM10 into soluble ephrinA2 fragments which are released into the blood in PCa patients. However, further research is required to fully uncover the underlying mechanism of the elevated serum ephrinA2 concentration in $\mathrm{PCa}$ remains.

PSA is a widely recognized and most important tumor marker for $\mathrm{PCa}$, which is applied in pathological diagnosis and observation of curative effects. Yet, in $\mathrm{BPH}$, urinary retention or inflammatory diseases, PSA level may exceed normal ranges. Therefore, a more sensitive and specific biomarker that distinguishes PCa from $\mathrm{BPH}$ is needed. In this research, we sought an alternative strategy to detect the presence of $\mathrm{PCa}$ via measuring serum exosomal ephrinA2 concentration and serum ephrinA2 levels in addition to serum PSA concentration. From the ROC curve, we found that the AUC of exosomal ephrinA2 was 0.9062 ( $\mathrm{CI}=0.8191-0.9933)$, while that of serum ephrinA2 was $0.7666(\mathrm{CI}=0.6284-0.9047)$ and that of serum PSA concentration was 0.6043 $(\mathrm{CI}=0.4586-0.7499)$. These observations imply that exosomal ephrinA2 level had a superior diagnostic value for clinical application compared to serum ephrinA2 or PSA concentrations. Yet, we acknowledge that more follow-up studies are required to validate this hypothesis and to support the use of exosomal ephrinA2 as a biomarker for PCa into clinical practice in future.

In summary, we not only found the presence of circulating ephrinA2 in the serum of PCa patients, but also show that exosomal ephrinA2 is an important component of the circulating ephrinA2. Although exosomal ephrinA2 concentrations are far less than the $\mathrm{Sn}$, the diagnostic efficiency of exosomal ephrinA2 was superior to the whole serum ephrinA2 in distinguishing PCa patients from BPH patients. These results illustrated that exosomal ephrinA2 is a potential biomarker for PCa and can be used to monitor the molecular progress for supervision the progression of PCa.

\section{Abbreviations}

AUC: area under curve;

$\mathrm{BPH}$ : benign prostatic hyperplasia;

CI: confidence interval;

GPI: glycosyl-phosphatidylinositol;

HCC: hepatocellular carcinoma;

PCa: prostate cancer;

PNB: prostate needle biopsies;

PVDF: polyvinylidene fluoride membrane;

ROC: receiver operating characteristic;

RPE: radical prostatectomy;

Sn: exosome-depleted supernatant;

TEM: Transmission Electron microscopy;

TMB: tetramethylbenzidine;

TNM: tumor-node-metastasis.

\section{Acknowledgements}

We are grateful to Jiangsu Key Laboratory of Anesthesia and Analgesia Application Technology for the technical assistance. This study was supported by the Grant from the Department of Science \& Technology, Xuzhou, Jiangsu, China (No. KC16SL108), the Jiangsu Provincial Medical Youth Talent (No. QNRC2016781), the Natural Science Foundation of Jiangsu Province (No. BK20160233, No. BK20140228).

\section{Ethical approval}

The samples from human in this study were obtained in accordance with the ethical standards of the ethics committee of the affiliated Hospital of Xuzhou Medical University and the Institutional Review Board and the 1964 Helsinki Declaration and its later amendments. 


\section{Competing Interests}

The authors have declared that no competing interest exists.

\section{References}

[1] Siegel RL, Miller KD, Jemal A. Cancer statistics, 2016. CA Cancer J Clin. 2016. 66(1): 7-30.

[2] Schröder FH, Hugosson J, Roobol MJ, et al. Screening and prostate-cancer mortality in a randomized European study. N Engl J Med. 2009. 360(13): 1320-8.

[3] Hugosson J, Carlsson S, Aus G, et al. Mortality results from the Göteborg randomised population-based prostate-cancer screening trial. Lancet Oncol. 2010. 11(8): 725-32.

[4] Noberini R, de la Torre E R, Pasquale EB. Profiling Eph receptor expression in cells and tissues: a targeted mass spectrometry approach. Cell Adh Migr. 2012. 6(2): 102-12.

[5] Jurney WM, Gallo G, Letourneau PC, McLoon SC. Rac1-mediated endocytosis during ephrin-A2- and semaphorin 3A-induced growth cone collapse. J Neurosci. 2002. 22(14): 6019-28.

[6] Fox BP, Kandpal RP. Invasiveness of breast carcinoma cells and transcript profile: Eph receptors and ephrin ligands as molecular markers of potential diagnostic and prognostic application. Biochem Biophys Res Commun. 2004. 318(4): 882-92.

[7] Feng YX, Zhao JS, Li JJ, et al. Liver cancer: EphrinA2 promotes tumorigenicity through Rac1/Akt/NF-kappaB signaling pathway. Hepatology. 2010. 51(2): 535-44.

[8] Hafner C, Schmitz G, Meyer S, et al. Differential gene expression of Eph receptors and ephrins in benign human tissues and cancers. Clin Chem. 2004. 50(3): 490-9.

[9] Li S, Wu Z, Chen $\mathrm{Y}$, et al. Diagnostic and prognostic value of tissue and circulating levels of Ephrin-A2 in prostate cancer. Tumour Biol. 2016. 37(4): 5365-74.

[10] Sun W, Zhao C, Li Y, et al. Osteoclast-derived microRNA-containing exosomes selectively inhibit osteoblast activity. Cell Discov. 2016. 2: 16015.

[11] Saylor PJ. Bone targeted therapies for the prevention of skeletal morbidity in men with prostate cancer. Asian J Androl. 2014. 16(3): 341-7.

[12] Théry C, Amigorena S, Raposo G, Clayton A. Isolation and characterization of exosomes from cell culture supernatants and biological fluids. Curr Protoc Cell Biol. 2006. Chapter 3: Unit 3.22.

[13] Théry C, Zitvogel L, Amigorena S. Exosomes: composition, biogenesis and function. Nat Rev Immunol. 2002. 2(8): 569-79.

[14] Caby MP, Lankar D, Vincendeau-Scherrer C, Raposo G, Bonnerot C. Exosomal-like vesicles are present in human blood plasma. Int Immunol. 2005. 17(7): 879-87.

[15] Keller S, Sanderson MP, Stoeck A, Altevogt P. Exosomes: from biogenesis and secretion to biological function. Immunol Lett. 2006. 107(2): 102-8.

[16] Ogawa Y, Kanai-Azuma M, Akimoto Y, Kawakami H, Yanoshita R. Exosome-like vesicles with dipeptidyl peptidase IV in human saliva. Biol Pharm Bull. 2008. 31(6): 1059-62.

[17] Zhao Y, Chen $\mathrm{K}, \mathrm{Li} \mathrm{H}, \mathrm{Wu} \mathrm{H}$. Effect of $\mathrm{pH}$ on the isolation of urinary exosome. Int Urol Nephrol. 2017,49(1):165-9.

[18] Wu WK, Law PT, Lee CW, et al. MicroRNA in colorectal cancer: from benchtop to bedside. Carcinogenesis. 2011. 32(3): 247-53.

[19] Choi DS, Park JO, Jang SC, et al. Proteomic analysis of microvesicles derived from human colorectal cancer ascites. Proteomics. 2011. 11(13): 2745-51.

[20] Gong J, Körner R, Gaitanos L, Klein R. Exosomes mediate cell contact-independent ephrin-Eph signaling during axon guidance. J Cell Biol. 2016. 214(1): 35-44.

[21] Turay D, Khan S, Diaz OCJ, et al. Proteomic Profiling of Serum-Derived Exosomes from Ethnically Diverse Prostate Cancer Patients. Cancer Invest. 2016. 34(1): 1-11

[22] Hattori M, Osterfield M, Flanagan JG. Regulated cleavage of a contact-mediated axon repellent. Science. 2000. 289(5483): 1360-5.

[23] Janes PW, Saha N, Barton WA, et al. Adam meets Eph: an ADAM substrate recognition module acts as a molecular switch for ephrin cleavage in trans. Cell. 2005. 123(2): 291-304. 